\title{
The ST131 Escherichia coli H22 subclone from human intestinal microbiota: Comparison of genomic and phenotypic traits with those of the globally successful H30 subclone
}

Marie-Hélène Nicolas-Chanoine ${ }^{1,2,3^{*}}$, Marie Petitjean ${ }^{4,5}$, Azucena Mora ${ }^{6}$, Noémie Mayer ${ }^{1}$, Jean-Philippe Lavigne ${ }^{7,8}$, Olivier Boulet ${ }^{9}$, Véronique Leflon-Guibout ${ }^{1}$, Jorge Blanco ${ }^{6}$ and Didier Hocquet ${ }^{4,5}$

\begin{abstract}
Background: In 2006, we found healthy subjects carrying ST131 Escherichia coli in their intestinal microbiota consisting of two populations: a subdominant population of fluoroquinolone-resistant $E$. coli belonging to subclone H3O (H30-R or subclade C1), the current worldwide dominant ST131 subclone, and a dominant E. coli population composed of antibiotic-susceptible E. coli belonging to subclone H22 (clade B), the precursor of subclone H30. We sequenced the whole genome of fecal H22 strain S250, compared it to the genomes of ExPEC ST131 H30-Rx strain JJ1886 and commensal ST131 H41 strain SE15, sought the H22-H30 genomic differences in our fecal strains and assessed their phenotypic consequences.

Results: We detected 173 genes found in the Virulence Factor Database, of which 148 were shared by the three ST131 genomes, whereas some were genome-specific, notably those allowing determination of virotype (D for S250 and C for JJ1886). We found three sequences of the FimH site involved in adhesion: two in S250 and SE15 close and identical, respectively, to that previously reported to confer strong intestinal adhesion, and one in JJ1886, corresponding to that commonly present in uropathogenic E. coli. Among the genes involved in sugar metabolism, one encoding a gluconate kinase lacked in S250 and JJ1886. Although this gene was also absent in both our fecal H22 and H3O-R strains, H22 strains showed a higher capacity to grow in minimal medium with gluconate. Among the genes involved in gluconate metabolism, only the ghrB gene differed between S250/H22 and JJ1886/H30-R strains, resulting in different gluconate reductases. Of the genes involved in biofilm formation, two were absent in the three genomes and one, fimB, in the JJ1886 genome. Our fecal H30-R strains lacking intact fimB displayed delayed biofilm formation relative to our fecal H22 strains. The H22 strains differed by subclade B type and plasmid content, whereas the H30-R strains were identical.

Conclusions: Phenotypic analysis of our fecal strains based on observed genomic differences between S250 and JJ1886 strains suggests the presence of traits related to bacterial commensalism in our H22 strains and traits commonly found in uropathogenic E. coli in our H30-R strains.
\end{abstract}

Keywords: E. coli ST131, H22 genome, Sugar metabolism, Mannose-binding FimH region, Biofilm, Subclades B, Plasmid replicons

\footnotetext{
* Correspondence: mhn.chanoine@aphp.fr

${ }^{1}$ Service de Microbiologie, Hôpital Beaujon, AP-HP, Clichy, France

${ }^{2}$ Faculté de Médecine Paris Diderot, Paris, France

Full list of author information is available at the end of the article
} 


\section{Background}

Phylogenetic group B2, sequence type (ST) 131 Escherichia coli has been a worldwide dominant human extraintestinal pathogenic E. coli (ExPEC) since the beginning of the 2000s, and is among those resistant to fluoroquinolones and/or producing the extended-spectrum $\beta$ lactamase (ESBL) CTX-M-15 [1]. Its dominance was shown to be driven by the expansion of a subclone harboring type 1 fimbriae-encoding fimH allele 30 (subclone H30), comprised of mostly strains resistant to fluoroquinolones (H30-R) [2]. Within this subclone, a subgroup called H30-Rx, mostly comprising strains resistant to fluoroquinolones and producing CTX-M-15, has quickly emerged and disseminated [2]. The evolutionary history of clone ST131 revealed that before the emergence and dissemination of subclone H30 (also called clade $\mathrm{C}$ ), the ST131 population consisted of mostly two subclones called $H 22$ (clade B) and $H 41$ (clade A), with subclone $H 22$ comprised of mostly fluoroquinolone-susceptible isolates [3-5]. It also revealed that subclone $H 22$ was the precursor of subclone $H 30$ and that separate F-type plasmids have shaped the evolution of subclone $H 30$ [2, 3, 5, 6]. The most recent ST131 E. coli phylogenetic reconstruction carried out by Ben Zakour et al. using 3779 non-recombinant single nucleotide polymorphisms (SNP) found in the high quality genomes of 172 clade $B$ and $C$ strains, identified five $B$ subclades, for which the independent evolutionary trajectories are characterized by successive insertions and a recombination from ancestral $H 22$ (subclade B1) [7]. Subclade B2 is characterized by a Flag- 2 locus insertion, subclade B3 by a Flag-2 locus insertion and par-C2 recombination, subclade B4 by Flag-2 locus and GI-PheV insertions, and subclade B5 by Flag-2 locus and Phi3 insertions. Other insertions, notably that of GI-PheV, recombination events, and mutations in $g y r A$ and parC occurred within strains of subclade B5, resulting in their evolution to clade $\mathrm{C}$ and subclades $\mathrm{C} 1$ (H30-R) and C2 $(H 30-\mathrm{Rx})$. Epidemiologically, the 172 isolates consisted of mostly those from North America obtained between 1948 and 2011, with most collected between 2000 and 2010, irrespective of the clade and subclade types. Since the first description of the fimH lineage in 2013 [3], the fimH type has been found in several epidemiological studies published on ExPEC or fecal ST131 isolates [8-17]. However, these have mostly concerned fluoroquinoloneresistant and /or ESBL-producing isolates. In 2006, we retrieved non-ESBL-producing ST131 E. coli isolates, susceptible or resistant to fluoroquinolones, from the intestinal microbiota of $7 \%$ of healthy subjects living in the Paris area [18]. The fluoroquinolone-resistant isolates accounted for a subdominant $E$. coli population in four independent healthy subjects, whereas those susceptible to fluoroquinolones accounted for the dominant $E$. coli population in three other independent subjects. The latter finding strongly suggests that ST131 E. coli is part of the normal intestinal microbiota of humans. In the present study, we aimed to determine whether these isolates belong to different $H$ subclones, based on their fluoroquinolonesusceptibility pattern. The dominant fluoroquinolonesusceptible ST131 E. coli populations belonged to subclone $H 22$. Assembled and annotated genomes of H22 strains were not available when we performed the present study. Thus, we first sequenced the whole genome of one of our commensal H22 strains (S250) and compared it to the genome of two reference ST131 strains: the ExPEC H30-Rx strain JJ1886 and the commensal H41 strain SE15. This genome comparison focused on virulence factor (VF)-encoding genes and those encoding processes or structures (sugar metabolism, biofilm formation, and the FimH mannose-binding site) known to be involved in the adaptation of the bacteria to different environments including human intestine. The four fluoroquinolone-resistant fecal ST131 isolates were $H 30-\mathrm{R}$ strains. Thus, we compared them to the three fecal H22 strains considering the genomic differences identified between H22 strain S250 and H30-Rx strain JJ1886, and analyzed the phenotypic impact of some of these differences.

\section{Methods}

\section{Bacterial strains}

The seven fecal ST131 isolates (strains 02, 39, 183, 187, 196, 208, and S250) obtained from the intestinal microbiota of seven healthy subjects living in the Paris area in 2006 were included in the study. They display serotype O25:H4, do not produce ESBL, and are either susceptible (196, 208 and S250) or resistant (02, 39, 183 and 187) to fluoroquinolones [18]. The fluoroquinoloneresistant strains were retrieved by plating the feces of healthy subjects on nalidixic acid-containing plates. The fluoroquinolone-susceptible strains accounted for the dominant E. coli population of three subjects. We previously assessed the virulence potential of strain S250 in the Caenorhabditis elegans and zebrafish models and analyzed its genome by optical mapping. This strain had a level of virulence similar to that of the multidrug resistant ST131 isolates, with which it shared $86 \%$ genome similarity [19]. We also included the E. coli K-12 MG1655 reference strain in the study as a control in the experiments assessing the use of gluconate as the sole source of carbon.

\section{fimH type}

fimH typing was based on the internal 489-nucleotide (nt) sequence of the fimH gene as previously described [20]. 


\section{Antibiotic susceptibility}

Antibiotic susceptibility was determined by the agar diffusion method and interpreted following the 2015 EUCAST recommendations (www.eucast.org). The following antibiotics were tested: amoxicillin, amoxicillin + clavulanic acid, ciprofloxacin, gentamicin, amikacin, cotrimoxazole and fosfomycin.

\section{Molecular analysis of resistance mechanisms}

The genes encoding resistance to amoxicillin (TEM and SHV enzymes) were identified by PCR and sequencing methods as previously described [21]. The qnr genes encoding plasmid-mediated resistance to fluoroquinolones were tested and the quinolone resistance determining region (QRDR) of the genes $\operatorname{gyr} A, \operatorname{gyr} B, \operatorname{parC}$ and $\operatorname{parE}$ amplified and sequenced using methods previously described [21-23]. The QRDRs of our strains were compared with those of the fluoroquinolone-susceptible reference strain E. coli K-12 MG1655 [GenBank: CP014225.1] and the allelic gyrA and parC profiles were compared with those previously described for ST131 isolates [3].

\section{Sequencing and analysis of the whole genome of $\mathrm{H} 22$ strain S250}

The complete genomic sequence was determined for H22 strain S250. Total DNA was extracted using the Qiagen Blood \& Cell Culture DNA Mini Kit (Qiagen, Courtaboeuf, France). Libraries were constructed using Nextera technology and sequenced on an Illumina HiSeq-2000 using a $2 \times 100$ nucleotides (nt) paired-end strategy. All reads were processed to remove low quality or artefactual nucleotides, using sequentially sickle (www.github.com/najoshi/sickle), AlienTrimmer [24] and fqDuplicate (ftp.pasteur.fr/pub/gensoft/projects/fqtools). Read pairs were assembled using clc_assembler from the CLC Genomics Workbench analysis package (www.clcbio.com/products/clc-genomics-workbench). All contigs of $\geq 500$ nt were reordered and reoriented, using the genomic sequence of strain $E$. coli K-12 MG1655 as a reference, with Mauve Contig Mover [25]. The reordered contigs were analyzed. The genome of H22 strain S250 was compared to that of two reference ST131 strains, JJ1886 (H30-Rx) [GenBank: CP006784.1] and SE15 (H41) [GenBank: AP009378.1], focusing on VF-encoding genes and genes involved in sugar metabolism, biofilm formation, and methylation. We downloaded VFs (www.mgc.ac.cn/VFs/) available from the Virulence Factor Database (VFDB) and searched the three genomes for their presence using Prodigal v2.6.1 [26] and clustered them at $90 \%$ identity using CD-hit v4.6 [27]. We extracted the sequences of the genes involved in biofilm formation in strain $E$. coli $\mathrm{K} 12$ BW25113 [28] from its genome [GenBank: CP009273.1] and clustered them at 90\% identity with CD-hit. We assessed the percentage identity between the genes from E. coli K12 BW25113 and the three studied genomes. Using the NBCI basic local alignment search tool (BLAST), we blasted genes from E. coli K12 MG1655 against the S250, JJ1886, and SE15 genomes to verify the possible absence of any genes involved in biofilm formation. We then searched the genomes of ST131 strains S250, JJ1886, and SE15 for genes involved in sugar metabolism as defined by Maltby et al. [29] in commensal strains E. coli HS [GenBank: CP000802.1] and E. coli Nissle 1917 [GenBank: CP007799.1] using BLAST. Moreover, we also searched for all the genes involved in gluconate metabolism (main pathway: gntR, gntT, gntU, gntP, gntK; secondary pathway: idnT, idnDOTR; gene $i d n K$ (gntV) which plays a role in the two pathways; the Entner-Doudoroff pathway: edd and eda; additional genes involved in other pathways: $k d g T, k d g K, g n d, t k r A$, $g h r B, k d u D, d k g A$ and $d k g B$ ) [30], as well as the 100 base pairs (bps) upstream of the start codon of each, in the genome of strains S250 and JJ1886. The nucleotide sequence of the $100 \mathrm{bp}$-upstream regions and the sequences of the deduced proteins for each gene of the strains S250 and JJ1886 were compared. We also searched for genes encoding methyltransferases in ST131 strain EC958 [GenBank: HG941718.1] [31] in the genome of ST131 strains S250, JJ1886, and SE15 using BLAST.

\section{Subclade B and clade C typing}

According to the work of Ben Zakour et al. [7], we determined the subclade B type of $H 22$ strain S250 and the clade $\mathrm{C}$ type of strain JJ1886 by direct blasting of the genes of the Flag-2 locus (Flag-2 locus from strain E. coli 042 [EMBL: CR 753847]), Phi3 (from strain EC958), and GI-PheV (from strain JJ1886) against the genome of strains S250 and JJI886. We then determined the type of subclade B displayed by strain S250 and the two other fecal $H 22$ strains by PCR using primers specific for the Flag-2 locus, Phi3, and GI-PheV (Additional file 1: Table S1) and our H30-R fecal strains as a positive control.

\section{Plasmid content}

The plasmid content of $H 22$ strain S250 was determined using the PlasmidFinder system [32] and the FII, FIA, and FIB formula of the detected IncF plasmid by PCRbased replicon typing (http://pubmlst.org/plasmid/). The latter method was also applied to the two remaining fecal $H 22$ strains and the four fecal H30-R strains.

\section{EXPEC status and virotype}

According to the study of Johnson and Adam, ExPEC status is defined by the presence of $\geq 2 \mathrm{VF}$ genes among the following genes: pap, sfa/focDE, afa/draBC, iutA, 
and kpsMT II [33]. As the latter gene is not included in the VFDB, we searched for it by blasting the kpsMT II genes [GenBank: X53819] against the S250, JJ1886, and SE15 genomes. Based on the study of Blanco et al., the major virotypes of E.coli ST131 are defined using four genes as follows: virotype $\mathrm{A}=a f a \mathrm{FM} 955459^{+}$iroN $N^{-} i b e A$ ${ }^{-}$sat $^{+/}$, virotype $\mathrm{B}=$ afaFM955459- iroN $^{+}$ibe $A^{-}$sat $^{+/-}$, virotype $\mathrm{C}=a f a \mathrm{FM} 955459^{-}$iroN $^{-} i b e A^{-}$sat $^{+}$, and viro-

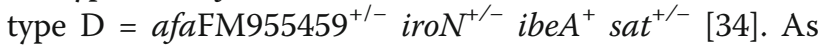
afaFM955459 is not included in the VFDB, we searched for it by blasting the afaFM955459 operon [EMBL: FM955459] against the S250, JJ1886, and SE15 genomes [35]. Then, classic multiplex PCR was used to search for genes encoding ExPEC-associated VFs (Additional file 2: Table S2) [34] in the seven fecal strains to confirm the results provided by the direct genome analysis of strain S250 and to determine the VF profile, virotype, and ExPEC status of the six remaining fecal strains.

\section{FimH structure}

According to the study of Sokurenko et al., the amino acid variations observed within the sequence of the adhesin, FimH, of type 1 fimbriae, result in different levels of binding to mono-mannose $\left(\mathrm{M}_{1}\right)$ structures, whereas they have no impact on the normal high level of binding to tri-mannose $\left(\mathrm{M}_{3}\right)$ structures [36]. By measuring the ratio of $\mathrm{M}_{1} / \mathrm{M}_{3}$ binding in different experimental models, they defined low $M_{1}$-binding $\left(M_{1} / M_{3}<0.1\right)$ and high $\mathrm{M}_{1}$-binding $\left(\mathrm{M}_{1} / \mathrm{M}_{3}>0.90\right)$ FimH phenotypes and showed that they are related to specific FimH sequences [37]. Therefore, the deduced protein sequence of FimH of $H 22$ strain S250 was compared with that of H30-Rx ST131 strain JJ1886 [GenBank: AGY86963.1], H41 ST131 strain SE15 [GenBank: BAI57801.1], phylogroup A E. coli strain MG1655 [GenBank: AMC97175.1], and two other phylogroup B2 strains: ST73 strain CFT073 [GenBank: AAN83822.1] and ST131 uk_P46212 [GenBank: ALT52319.1]. The deduced protein sequence of FimH was then characterized for the six remaining fecal strains by using primers for which the sequences have been previously published [20].

\section{Amplification and sequencing of the idnK (gntV) and ghrB genes of the seven fecal strains and growth with gluconate as the sole carbon source}

The idnk (gntV) gene, encoding a thermosensitive Dgluconate kinase, and the $g h r B$ gene, encoding a gluconate reductase, were amplified with primers indicated in Additional file 1: Table S1. Additional primers were used to sequence the $g h r B$ gene (Additional file 1: Table S1). We evaluated the ability of the seven strains to grow in the presence of gluconate as the sole carbon source, as previously described [38]. Experiments were conducted three times independently and all incubations were performed overnight at $37{ }^{\circ} \mathrm{C}$ with shaking (150 rpm). Briefly, bacteria were first cultured in Mueller Hinton broth before washing twice in minimal media M63 [15 mM $\left(\mathrm{NH}_{4}\right)_{2} \mathrm{SO}_{4} ; 100 \mathrm{mM} \quad \mathrm{KH}_{2} \mathrm{PO}_{4} ; 0.002 \mathrm{mM}$ $\left.\mathrm{FeSO}_{4}\left(7 \mathrm{H}_{2} \mathrm{O}\right)\right]$. We inoculated $5 \mathrm{ml}$ of $\mathrm{M} 63$ supplemented with $0.2 \%$ glucose (Sigma-Aldrich, France) with $10 \mu \mathrm{l}$ of the washed bacteria. One $\mathrm{ml}$ of this culture was washed twice in M63. We transferred $10 \mu \mathrm{l}$ of this washed culture into $5 \mathrm{ml}$ of M63 with $0.2 \%$ gluconate (Sigma-Aldrich, Saint-Quentin Fallavier, France). The cultures were then adjusted to 0.002 at $\mathrm{OD}_{600}$ in $30 \mathrm{ml}$ fresh M63 with $0.2 \%$ gluconate and incubated at $37{ }^{\circ} \mathrm{C}$ with shaking $(150 \mathrm{rpm})$. We estimated the bacterial growth after $48 \mathrm{~h}$ by measuring the $\mathrm{OD}_{600}$ [39]. Tukey's test was used for intergroup comparisons and R software for statistical analyses. $P$ values $<0.01$ were considered to be statistically significant.

\section{Amplification and sequencing of the $\operatorname{fim} B$ gene and kinetics of biofilm formation in the seven fecal strains}

The $\operatorname{fim} B$ gene was amplified and sequenced with the primers indicated in Additional file 1: Table S1. The kinetics of early biofilm formation was assessed using the BioFilm Ring Test $^{\circ}$ (BioFilm Control, Saint Beauzire, France), as described [40]. Briefly, standardized bacterial cultures were incubated at $37{ }^{\circ} \mathrm{C}$ in a 96 -well microtiter plate in the presence of magnetic beads. At various time points, the plates were placed onto a magnetic test block and put in the reader. The images of each well before and after magnetic attraction were analyzed using BioFilm Control software that gives a BioFilm Index (BFI). The BFI was converted into the proportion of immobilized beads relative to a reference condition (\% $\mathrm{RBI})$ using the formula: \% RBI $=\sqrt{ }\left[\left(1-\left(\mathrm{BFI}_{\text {assay }}-\mathrm{BFI}_{\min }\right) /\right.\right.$ $\left.\left(\mathrm{BFI}_{\text {control }}-\mathrm{BFI}_{\text {min }}\right)\right] \times 100$, where $\mathrm{BFI}_{\text {assay }}$ is the $\mathrm{BFI}$ of the tested strain, $\mathrm{BFI}_{\text {control }}$ is the $\mathrm{BFI}$ of the control, corresponding to the maximum $\mathrm{BFI}$, and $\mathrm{BFI}_{\min }$ is the minimal observed BFI when all the beads are blocked. The more RBI approaches a value of 1 , the more the biofilm is fully formed (beads are immobilized). Three experiments were performed in duplicated per strain and per incubation time. The kinetics of biofilm formation were compared using a two-way ANOVA followed by Dunnett's multiple comparisons test.

\section{Results}

fimH type, antibiotic susceptibility, molecular analysis of resistance mechanisms, and allelic profiles of the gyrA and parC genes

The three fluoroquinolone-susceptible strains (S250, 208 and 196) were of the fimH 22 type. All but one were susceptible to all of the antibiotics tested (Table 1). H22 strain 196 was resistant to both amoxicillin and cotrimoxazole. The four fluoroquinolone-resistant strains 
Table 1 Characterization of the seven fecal strains of E. coli ST131

\begin{tabular}{|c|c|c|c|c|c|c|c|c|c|c|c|c|c|c|c|c|}
\hline \multirow{2}{*}{$\begin{array}{l}\text { Strain/ FimH } \\
\text { type }\end{array}$} & \multicolumn{7}{|c|}{ Susceptibility } & \multirow[t]{2}{*}{$\beta$-lactamase } & \multicolumn{3}{|c|}{$\begin{array}{l}\text { Allele type } \\
\text { (amino acid substitution) }\end{array}$} & \multirow[t]{2}{*}{ Flag-2 } & \multirow[t]{2}{*}{ Phi3 } & \multirow[t]{2}{*}{ Gl-PheV } & \multirow[t]{2}{*}{ Subclade } & \multirow{2}{*}{$\begin{array}{l}\text { IncF } \\
\text { plasmid } \\
\text { replicon }\end{array}$} \\
\hline & AMX & AMC & CIP & GEN & AMK & SXT & FOS & & gyrA & parC & parE & & & & & \\
\hline $\mathrm{S} 250 / \mathrm{H} 22$ & $\mathrm{~S}$ & $\mathrm{~S}$ & $\mathrm{~S}$ & $\mathrm{~S}$ & $\mathrm{~S}$ & $S$ & $S$ & - & $1 a$ & 1 & $\begin{array}{l}\text { NA } \\
\text { (1529L) }\end{array}$ & - & - & - & B1 & F89:A-B62 \\
\hline 208/H22 & $S$ & $S$ & S & S & $S$ & $S$ & $S$ & - & $1 a$ & 1 & $\begin{array}{l}\text { NA } \\
(1529 L)\end{array}$ & + & - & - & B2 & - \\
\hline $196 / H 22$ & $\mathrm{R}$ & $S$ & S & S & $S$ & $\mathrm{R}$ & S & TEM-1 & $1 a$ & 1 & $\begin{array}{l}\text { NA } \\
(1529 L)\end{array}$ & + & - & + & B4 & F24:A-:B6 \\
\hline 187/H30 & $\mathrm{R}$ & $S$ & R & S & S & $S$ & $S$ & TEM-1 & $\begin{array}{l}1 \mathrm{AB} \\
(\mathrm{S} 83 \mathrm{~L} / \mathrm{D} 87 \mathrm{~N})\end{array}$ & $\begin{array}{l}1 \mathrm{aAB} \\
(\mathrm{S} 80 \mathrm{l} / \mathrm{E} 84 \mathrm{~V})\end{array}$ & $\begin{array}{l}\text { NA } \\
(1529 L)\end{array}$ & + & + & + & $\mathrm{C} 1$ & F1:A2:B20 \\
\hline 183/H30 & $\mathrm{R}$ & $S$ & R & S & S & $S$ & $S$ & TEM-1 & $\begin{array}{l}1 \mathrm{AB} \\
(\mathrm{S} 83 \mathrm{~L} / \mathrm{D} 87 \mathrm{~N})\end{array}$ & $\begin{array}{l}\text { 1aAB } \\
\text { (S80I/E84V) }\end{array}$ & $\begin{array}{l}\text { NA } \\
(1529 L)\end{array}$ & + & + & + & $\mathrm{C} 1$ & $\mathrm{~F} 1: \mathrm{A} 2: \mathrm{B} 20$ \\
\hline 39/H30 & $\mathrm{R}$ & $S$ & R & S & S & $S$ & S & TEM-1 & $\begin{array}{l}1 \mathrm{AB} \\
(\mathrm{S} 83 \mathrm{~L} / \mathrm{D} 87 \mathrm{~N})\end{array}$ & $\begin{array}{l}1 \mathrm{aAB} \\
\text { (S80I/E84V) }\end{array}$ & $\begin{array}{l}\text { NA } \\
\text { (I529L) }\end{array}$ & + & + & + & $\mathrm{C} 1$ & $\mathrm{~F} 1: \mathrm{A} 2: \mathrm{B} 20$ \\
\hline $02 / H 30$ & $\mathrm{R}$ & $S$ & R & S & S & S & $S$ & TEM-1 & $\begin{array}{l}1 \mathrm{AB} \\
(\mathrm{S} 83 \mathrm{~L} / \mathrm{D} 87 \mathrm{~N})\end{array}$ & $\begin{array}{l}\text { 1aAB } \\
\text { (S801/E84V) }\end{array}$ & $\begin{array}{l}\text { NA } \\
(1529 L)\end{array}$ & + & + & + & $\mathrm{C} 1$ & $\mathrm{~F} 1: \mathrm{A} 2: \mathrm{B} 20$ \\
\hline
\end{tabular}

according to reference [3], AMX amoxicillin, AMC amoxicillin + clavulanic acid, CIP ciprofloxacin, GEN gentamicin, AMK amikacin, SXT cotrimoxazole, FOS fosfomycin, $R$ resistant, $S$ susceptible, - : absence, + : presence, $N A$ not available

(187, 183, 39 and 02), which were all resistant to amoxicillin but susceptible to the other antibiotics tested, were of the fimH30 type (H30-R) (Table 1$)$. QRDR nucleotide sequence analysis showed that the three $H 22$ strains displayed gyrA1a and parC1 alleles, whereas the four H30$\mathrm{R}$ strains displayed the gyr $\mathrm{A} \mathrm{AB}$ allele, encoding amino acid substitutions $\mathrm{S} 83 \mathrm{~L}$ and $\mathrm{D} 87 \mathrm{~N}$, and the parC1aAB allele, encoding amino acid substitutions S80I and E84V (Table 1). We found the substitution I529L in ParE in the seven fecal strains (Table 1). None of these strains harbored plasmid-mediated qnr genes. H22 196 and the four H30-R strains that were resistant to amoxicillin harbored a TEM-1-encoding gene (Table 1).

\section{Genomic and phenotypic characterization}

We assembled the whole genome sequence of the fecal H22 strain S250 into 50 contigs and analyzed and compared it with those of the ExPEC H30-Rx strain JJ1886 and the commensal H41 strain SE15.

\section{Subclade $B$ and $C$ type}

We were unable to find the genes composing the Flag-2 locus in the genome of $H 22$ strain S250 using BLAST. We only found a fragment of approximately $1700 \mathrm{bp}$ which was very similar to the end of the first gene, IfhA, and another of $769 \mathrm{bp}$ similar to the end to the last gene, lafU, of the Flag-2 locus (data not shown). We found neither Phi3 nor GI-PheV in the genome of H22strain S250, whereas we found them, as well as the Flag-2 locus, in the genome of JJ11886. The use of specific primers allowed us to confirm the absence of these genetic elements in $H 22$ strain 250 and to detect the Flag-2 locus in H22 strains 196 and 208, as well as GI-PheV in
H22 strain 196. These three genetic elements were amplified from our four $H$-30R strains (Table 1).

\section{VF-encoding genes}

Using a gene identity level of $\geq 90 \%$, a total of 173 genes among the 2520 Escherichia sp. VF-encoding genes of the VFDB was identified in the genomes of the three ST131 strains studied. H22 strain S250, H30-Rx strain JJ1886, and H41 strain SE15 had 160, 159, and 152 VFencoding genes, respectively. The three ST131 genomes shared 148 virulence genes (Fig. 1). Nine genes were found specifically in $H 22$ strain S250: the five iroBCDEN genes that encode proteins related to a catecholate siderophore, the three pixCDH genes encoding Pix pilus adhesion, and the ibeA gene involved in invasion of brain endothelium (Fig. 1). Ten genes were specifically found in H30-RX strain JJ1886: the four iucABCD and iutA genes encoding proteins involved in the binding and transport of iron, the pap1 and papX genes encoding pap operon regulatory proteins, the sat gene encoding a toxin, the $f l u$ gene encoding autotransporter protein Ag43a, and the iha gene encoding the adhesionsiderophore receptor. Three genes, including the fimB gene, were present in strains H22 S250 and H41 SE15, but not H30-RX strain JJ1886 (Fig. 1). The ECP_2810 gene, encoding a Val-Gly Repeats-related protein, was present in H41 strain SE15 and H30-RX strain JJ1886.

\section{Virotype and ExPEC status}

The VF genes identified in the VFDB would suggest virotype $\mathrm{D}\left(i b e A^{+}\right.$, iroN $N^{+}$, and $\left.s a t^{-}\right)$for strain S250 and virotype C $\left(s a t^{+}\right.$, $i b e A^{-}$, and $\left.i r o N^{-}\right)$for strain JJ1886. These virotypes were confirmed by the absence of the 


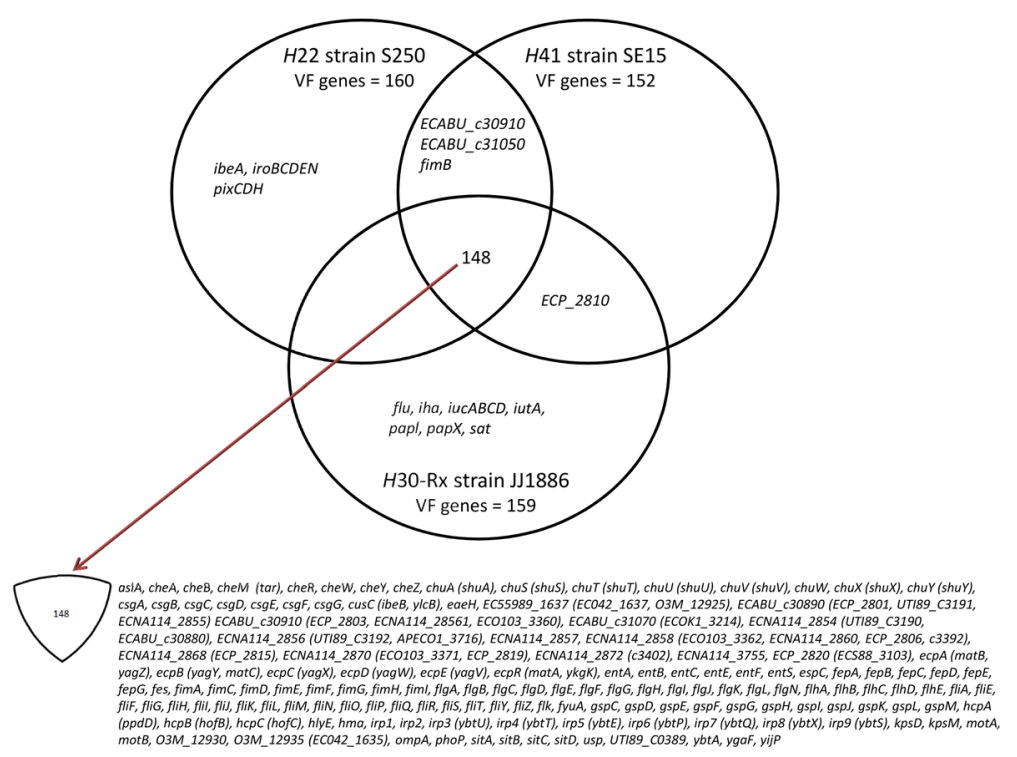

Fig. 1 List of genes found in the genomes of H22 strain S250, H30-Rx strain JJ1186 and, H41 strain SE15. Each strain is represented by a circle and each gene commonly found in the genome of two strains or in the genome of the three strains is indicated in the corresponding intersecting regions, whereas genes specific for each strain are indicated in the section of the circle not shared with another circle. The 148 genes present in the three strains are listed at the bottom of the figure

afaFM955459 operon in the genome of strains S250 and JJ1886, shown using BLAST. We were unable to determine a virotype for strain SE15, as none of the four genes were detected in the genome of this strain. Combining VFDB-based and BLAST analysis of the kpsMT II gene, we found that strains S250 and SE15 did not display an ExPEC status, as they harbored only one (kpsMT II) of the genes used to define this status. Multiplex PCR classically used to search for genes encoding ExPECassociated VF (Additional file 2: Table S2) to the seven fecal strains showed that the two remaining fecal $\mathrm{H} 22$ strains displayed virotype D, as strain S250, and the four fecal $H 30-\mathrm{R}$ strains virotype $\mathrm{C}$, as strain JJ1186 (Table 2). It also showed that only virotype $\mathrm{C} H 30-\mathrm{R}$ strain 39 displayed an ExPEC status related to the presence of the iutA and kpsMT II genes. We found 11 of the amplified VF genes (fimH, matB, pet, chuA, fyuA, irp2, sitA, traT, malX, usp and ompT) in all but one (strain $H 22$ 208) strain, five (F10 papA, iha, sat, iucD and iutA) in only $H 30-\mathrm{R}$ strains, and four ( $c d t$, iroN, iss and ibeA) in only $H 22$ strains. The number of amplified VF-encoding genes varied from 15 to 17 in $H 30-\mathrm{R}$ strains and 13 to 16 in $H 22$ strains.

\section{Deduced protein FimH}

As indicated in Fig. 2, the deduced protein sequence of adhesin FimH of representatives of H30-Rx ST131 E. coli (strains JJ1886 and uk P46212), H41 ST131 E. coli (strain SE15), H22 ST131 E. coli (strain S250), UPEC (ST73 strain CFT073), and E. coli K12 (strain MG1655) displayed amino acid differences at the positions previously shown to characterize different $\mathrm{M}_{1} / \mathrm{M}_{3}$ ratio phenotypes, contributing to different levels of colonization of different niches [37]. Thus, we identified the sequence -A27, N70 and S78 or N78 and S70, V163, and R166corresponding to the lower $M_{1} / M_{3}$ ratio (0.08-0.09) in

Table 2 Virulence factor-encoding genes, virotype, and EXPEC status of human fecal ST131 Escherichia coli H22 and H30 subclones

\begin{tabular}{|c|c|c|c|c|}
\hline Strain & $\mathrm{fimH}$ & Virulence factor-encoding gene & Virotype & ExPEC status \\
\hline S250 & $H 22$ & fimH, matB, pet, iroN, chuA, fyuA, irp2, sitA, kpsMII-K5, iss, trat, ibeA, malX, usp, ompT & D & - \\
\hline 208 & $H 22$ & fimH, matB, cdtB, pet, chuA, fyuA, irp2, sitA, kpsMII-K5, ibeA, malX, usp, ompT & $\mathrm{D}$ & - \\
\hline 196 & $H 22$ & fimH, matB, cdtB, pet, iroN, chuA, fyuA, irp2, sitA, kpsMII-K5, iss, traT, ibeA, malX, usp, ompT & $\mathrm{D}$ & - \\
\hline 187 & $H 30$ & fimH, F10 papA, iha, matB, sat, pet, iucD, iutA, chuA, fyuA, irp2, sitA, traT, malX, usp, ompT & C & - \\
\hline 183 & H30 & fimH, F10 papA, iha, matB, sat, pet, iucD, iutA, chuA, fyuA, irp2, sitA, traT, malX, usp, ompT & C & - \\
\hline 39 & $H 30$ & fimH, F10 papA, iha, matB, sat, pet, iucD, iutA, chuA, fyuA, irp2, sitA, kpsMII-K5, traT, malX, usp, ompT & C & + \\
\hline 02 & H30 & fimH, F10 papA, matB, sat, pet, iucD, iutA, chuA, fyuA, irp2, sitA, traT, malX, usp, ompT & C & - \\
\hline
\end{tabular}




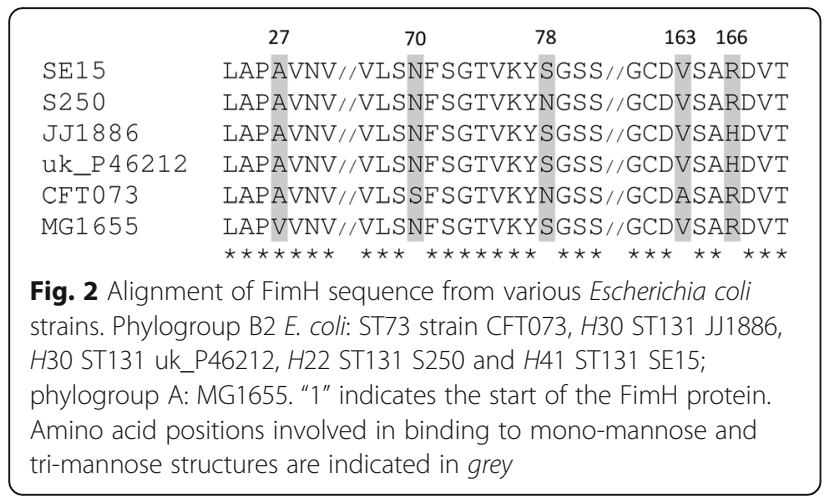

the study of Sokurenko et al. in strain SE15. We found the substitutions $\mathrm{A} 27 \mathrm{~V}$ and $\mathrm{V} 163 \mathrm{~A}$, that are each related to a ten-fold increase in the $M_{1} / M_{3}$ ratio in the Sokurenko et al., study in strain MG1655 and strain CFT073, respectively. We also found the substitution $\mathrm{R} 166 \mathrm{H}$ related to a $M_{1} / M_{3}$ ratio of 0.33 in the same study in H30-Rx strains JJ1886 and uk_P46212. H22 strain S250 displayed a sequence not published in the Sokurenko et al. study as residue $\mathrm{N}$ was present at both positions 70 and 78. However, we found none of the substitutions known to induce an increase in the $M_{1} / M_{3}$ ratio in the FimH sequence of strain S250. We found the same feature in the two other fecal $H 22$ strains, whereas the FimH sequence of our four H30-R strains was identical to that of H30-Rx strains JJ1886 and uk_ P46212 (data not shown).

\section{Genes involved in sugar metabolism and growth with gluconate as the sole carbon source}

All genes involved in sugar metabolism in commensal $E$. coli strains HS and Nissle were detected in the genome of H41 strain SE15, whereas one, the D-gluconate kinase-encoding idnK ( gntV) gene, was not detected in either strain H30-Rx JJ1886 or H22 S250 (Table 3). Its absence in our six remaining strains was revealed by the PCR-sequencing assay. Therefore, we assessed the ability of our strains to grow with gluconate as the sole carbon source in the absence of the $i d n K$ ( $g n t V$ ) gene and the presence of the gntK gene, which both transform D-gluconate to $6 \mathrm{P}$-gluconate. After $48 \mathrm{~h}$ of growth, the biomass of our H30-R strains was significantly lower than that for our $H 22$ strains (Tukey's test $p<0.01$ ) (Fig. 3). This unexpected difference led us to compare the genomes of strains H22 S250 and H30-Rx JJ1886 focusing on all the genes, other than the $i d n k(g \operatorname{tn} V)$ gene, involved in gluconate metabolism, as well as the $100 \mathrm{bp}$ upstream of the start codon of the operons or genes. All were present in the genome of strains S250 and JJ1886. The $100 \mathrm{bp}$ upstream of the start codon and the sequence of the deduced proteins showed 100\% identity (data not shown) between these two strains, except for the coding region of the $g h r B$ gene,
Table 3 Distribution of the genes involved in sugar metabolism in commensal Escherichia coli strains HS and Nissle [29] in the genomes of representative $\mathrm{H3O}, \mathrm{H} 22$, and $\mathrm{H} 41$ subclones of Escherichia coli ST131

\begin{tabular}{lllll}
\hline Commensal & Catabolized & H30 & H22 & H41 \\
strain/gene & sugar & J1886 & S250 & SE15 \\
\hline E. coli HS & & & & \\
araA & Arabinose & + & + & + \\
araB & Arabinose & + & + & + \\
araD & Arabinose & + & + & + \\
fuck & Fucose & + & + & + \\
galk & Galactose & + & + & + \\
gntK & Gluconate & + & + & + \\
idnK & Gluconate & - & - & + \\
lacZ & Lactose & + & + & + \\
manA & Mannose & + & + & + \\
nagE & N-acetylglucosamine & + & + & + \\
nanA & N-acetylneuraminate & + & + & + \\
nanT1 & N-acetylneuraminate & + & + & + \\
rbsK & Ribose & + & + & + \\
uxaC & Glucuronate & + & + & + \\
E. coli Nissle 1917 & & & & + \\
agaA & N-acetylgalactosamine & + & + & + \\
agaE & N-acetylgalactosamine & + & + & + \\
agaF & N-acetylgalactosamine & + & + & + \\
agaW & N-acetylgalactosamine & + & + & + \\
\hline
\end{tabular}

which showed a T968C substitution, resulting in amino acid substitution V323A in strain JJ1886. This substitution was confirmed in our four fecal H30-R strains, whereas V323 was identified in the two remaining $H 22$ fecal strains.

\section{Biofilm genotype and phenotype}

The expression of a wide panel of genes can influence biofilm formation [28]. We specifically searched for these genes in the genome of strains JJ1886, S250, and SE15 (Additional file 3: Table S3). Two genes of the biofilm gene panel, fliC and $f l i D$, were missing from the three tested genomes. We aligned the $f l i C$ and $f l i D$ genes from E. coli strain MG1655 onto the three genomes (JJ1886, S250 and SE15) to verify their absence and found deletions within $f l i C$ and the absence of $f l i D$ in the three strains. An intact version of the $\operatorname{fim} B$ gene was present in strains $H 22 \mathrm{~S} 250$ and $H 41 \mathrm{SE} 15$, but it was disrupted in H30-Rx JJ1886 due to the insertion of IS3like, as previously described in various $H 30$ strains [34, 41]. The difference in the structure of the fimB gene in strains H30-Rx JJ1886 and H22 250 led us to sequence the $\operatorname{fim} B$ gene of the remaining fecal strains. We found that the $\operatorname{fim} B$ nucleotide sequence in the four H30-R strains was identical to that of $H 30-\mathrm{Rx}$ strain JJ1886, 


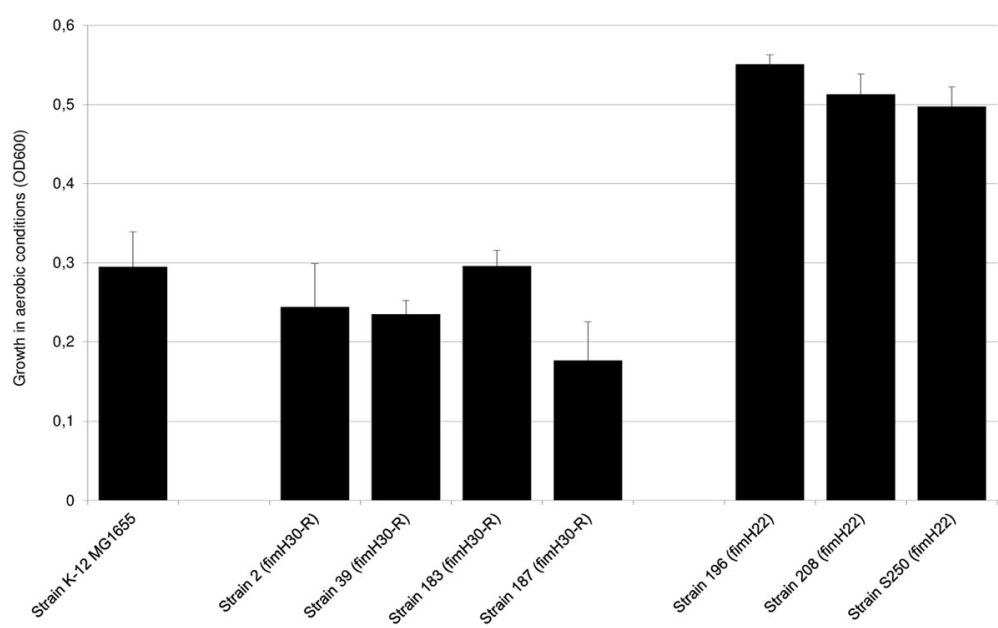

Fig. 3 Growth of human fecal subclone H30 and subclone H22 strains of ST131 Escherichia coli in minimal medium plus $0.2 \%$ gluconate. Strain growth was assessed by measuring the OD at $600 \mathrm{~nm}$ after $48 \mathrm{~h}$ of incubation. Bars represent the standard deviation obtained from three independent experiments

whereas that of the two remaining $H 22$ strains was identical to that of strain S250. Niba et al. previously reported that the deletion of $\mathrm{fliC}$ and/or $\mathrm{fliD}$ induces a substantial decrease in biofilm formation, whereas deletion of $\operatorname{fimB}$ results in in its near complete absence (Additional file 3: Table S3). We thus tested whether this fimB polymorphism affects biofilm formation. Kinetic measurements of early biofilm formation showed that the three $H 22$ strains formed biofilms significantly earlier $(p<0.0001)$ than the four H30-R strains (Fig. 4). Of note, H30-R strain 39 , which was the single fecal strain with an ExPEC status, showed significantly $(p<0.0001)$

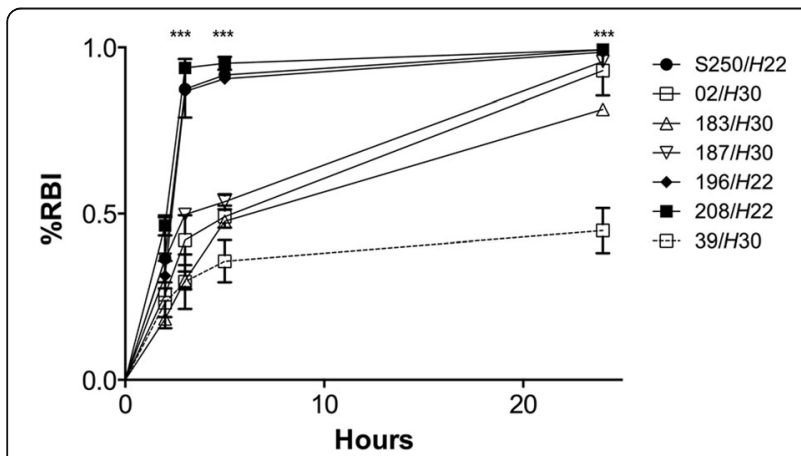

Fig. 4 Early biofilm formation by human fecal subclone $\mathrm{H} 30$ and subclone H22 strains of ST131 Escherichia coli. BioFilm Control ${ }^{\circledR}$ image analysis software was used and results are expressed as the proportion of immobilized beads relative to reference conditions (\% RBI) according to incubation time. Bars represent the standard deviation obtained from three independent experiments performed in duplicate. The biofilm is more fully formed (beads are immobilized) as RBI approaches a value of 1 . Significant differences between $\mathrm{H}_{22}$ and $\mathrm{H} 30$ using Dunnett's test are indicated by * $(p<0.05),{ }^{* *}(p<0.01)$ and ${ }^{* * *}(p<0.001)$ slower biofilm production (incomplete biofilm formation after $24 \mathrm{~h}$ ) than the other H30-R strains.

\section{Genes encoding methyltransferases}

We found all but one of the ten methyltransferases, previously identified in the genome of H30 ST131strain EC958 [31], in the genome of H30-Rx strain JJ1886 (Table 4). We found six in the genome of $H 41$ strain SE15 and only three in the genome of $H 22$ strain S250 (Table 4).

\section{Plasmid content}

We detected an IncFII plasmid in contig 4 and an IncFIB element in contig 41 of the S250 genome using PlasmidFinder. Using NCBI BLAST and the plasmid MSLT typing system, we identified the replicon alleles

Table 4 Distribution of the 10 methyltransferases previously described in H3O strain EC958, [31] in the genomes of representative $\mathrm{H} 30, \mathrm{H} 22$, and $\mathrm{H} 41$ subclones of Escherichia coli ST131

\begin{tabular}{llll} 
Methyltransferase (H30 EC958) & H30 JJ1886 & H22 S250 & H41 SE15 \\
\hline M.EcoMIV & + & - & - \\
M.EcoMV & + & - & - \\
M.EcoMDcm & + & + & + \\
EcoMIII & + & - & + \\
M.EcoMVI & + & + & + \\
M.EcoMDam & + & + & + \\
M.EcoMVII & - & - & - \\
M1.EcoMl & + & - & + \\
M2.EcoMl & + & - & + \\
M.EcoMII & + & - & - \\
Total number & 9 & 3 & 6
\end{tabular}

+: presence,-: absence 
F89 and B62 in the whole genome of strain S250. We confirmed this result for strain S250 by PCR-sequencing, and also obtained the following results for the six remaining fecal strains: F1:A2:B20 for the four H30-R strains, no IncF plasmid for $H 22$ strain 208, and F24:B6 for $H 22$ strain 196 (Table 1).

\section{Discussion}

CTX-M-15 producing, fluoroquinolone-resistant ST131 E. coli, which has been shown to be a worldwide human ExPEC since the beginning of the 2000s, has also been shown to colonize the human digestive tract [1]. In 2006, we found healthy subjects with a subdominant intestinal population of fluoroquinolone-resistant ST131 E. coli, as well as those with a dominant intestinal population of ST131 E. coli susceptible to fluoroquinolones [18]. The latter finding suggests that ST131 E. coli may be a human intestinal commensal. Here, we first showed that our three antibiotic-susceptible fecal ST131 E. coli strains displayed fimH22, whereas the four resistant to fluoroquinolones displayed fimH30 (H30-R). We thus centered our study on the comparison between these two groups of strains focusing on processes and structures involved in environmental adaptation, considering that subclone $H 22$ is the precursor of subclone $H 30$, which emerged at the end of 1990s [3, 7]. We first sequenced the whole genome of one of our fecal $H 22$ strains, strain S250, as there were no assembled and annotated genomes of $\mathrm{H} 22$ strains when we started our work. In contrast, there were sequenced whole genomes of representative multidrug resistant $H 30$ ExPECs, such as H30-RX strain JJ1886 and a commensal ST131 E. coli, strain SE15, belonging to another ST131 subclone, characterized by the allele fimH41. Comparison of the genomes of $H 22$ strain S250, H30-Rx strain JJ1886, and H41 strain SE15 showed high similarity in terms of the number and types of VF genes from the VFDB. This may explain the similar level of virulence that we found previously between $H 22$ strain S250 and an H30-Rx strain in the C. elegans model [19, 42]. Nevertheless, strains S250 and JJ1886 displayed two different virotypes: D for strain S250 and C for strain JJ1886. Moreover, strain S250 did not display the VF genes required for ExPEC status, whereas strain JJ1886 did [43]. There was also high similarity between the genes involved in biofilm formation in the three genomes [28], except for the fimB gene, which was disrupted in the H30-Rx strain JJ1886 and intact in both $H 22$ strain S250 and $H 41$ strain SE15. As the intact fimB gene was also absent from our fecal H30-R strains and present in our remaining fecal $H 22$ strains, we analyzed the kinetics of early biofilm formation in our seven fecal strains. Biofilm formation was significantly delayed in the H30-R strains. This is the first study to compare the formation of biofilms by $H 22$ and non-ESBL-producing $H 30-\mathrm{R}$ strains $[4,42,44-46]$. Further studies are required to verify the involvement of the fimB gene in the two different biofilm phenotypes. Comparison of the genes of the three genomes involved in the metabolism of various sugars showed the absence of one of the two genes encoding gluconate kinases in strains S250 and JJ1886. Although this gene was absent in both our fecal $H 22$ and H30-R strains, we found that $H 22$ strains had a higher capacity to grow in medium with gluconate as the sole carbon source. Thus, gluconate consumption may confer an advantage to $H 22$ strains for residing in the human intestine, as gluconate is a component of intestinal mucus [47]. Indeed, it has been shown that E. coli laboratory mutants with impaired growth on gluconate are less able to colonize the large intestine of mice [38]. Comparison of all genes, and their promoter regions, involved in the different pathways of gluconate metabolism showed one difference between strains S250 and JJ1886, namely a mutation leading to an amino acid substitution in the 2-keto-D-gluconate reductase GhrB in strain JJ1886. Although this genetic difference was found between the fecal $H 22$ and $H 30-\mathrm{R}$ strains, further studies are required to clarify the role of this mutation for the difference in the growth of the two strains when gluconate is the sole carbon source. Another difference we identified between strain S250 and strain JJ1886 concerned the sequence of the type 1 fimbriae FimH adhesin, shown by Sokurenko et al. to be involved in variations of the $M_{1} / M_{3}$ ratio associated with tissue tropism and the shift of bacterial adaptation from a commensal to pathological habitat $[36,37]$. The FimH sequence of strain JJ1886, which was also present in our H30-R fecal strains, corresponded to that identified by Sokurenko et al. in strains with a $\mathrm{M}_{1} /$ $M_{3}$ ratio $=0.33$. This sequence and $M_{1} / M_{3}$ ratio are commonly observed in uropathogenic strains [36, 37]. The FimH sequence identified in strain S250 and the other fecal $H 22$ strains was not found in the strains studied by Sokurenko et al. and was only found at a low frequency in those studied by Chen et al. [48]. This sequence showed none of the amino acid substitutions involved in the increase of the $M_{1} / M_{3}$ ratio. Thus it is likely to be associated with the lowest $M_{1} / M_{3}$ ratio shown by Sokurenko et al., which was associated with strong adhesion to intestinal cells [36, 37]. Altogether, although the $H 22$ and $H 30-\mathrm{R}$ strains investigated in this work were all isolated from the digestive tract of healthy subjects, only $H 22$ strains displayed properties previously shown to be associated with human intestinal commensalism, including competition for nutrients in the intestine (gluconate use) and a high intestinal adhesiveness [49].

We sought to confirm the genetic differences identified here between the lineages $H 22$ and $H 30$ with the 
whole genome sequences of ST131 isolates recently deposited on NCBI databases (i.e. $28 H 22$ strains, $9 H 30$ strains, see Additional file 4: Table S4). As expected, we retrieved all but one of these genetic differences in this wider $H 22$ and $H 30$ strain collection. Indeed, the gluconate reductase-encoding $g h r B$ gene in two $H 22$ clinical isolates (strain JJ1897 and strain GN02448 from the bioproject PRJNA290784: Additional file 4: Table S4) displayed the same sequence as in all $H 30$ strains and not that found in the other $H 22$ strains. Of note, these two strains possessed Phi3 and the par-Cla variant, the two evolutionary markers present in the $H 22$ "intermediate" strain group $\mathrm{B} 0 / \mathrm{C} 0$ described by Ben Zakour et al. [7]. The intermediate status was based on the SNP pattern of $H 22$ strains which progressively acquired clade $\mathrm{C}$-defining point mutations.

The last comparison made between the S250, JJ1886 and SE15 genomes concerned the genes encoding the 10 methyltransferases previously identified in the genome of H30-Rx ST131strain EC958 [31]. We found nine of these genes in strain JJ1886, six in strain SE15, and only three in strain S250. Forde et al. showed that six of the 10 genes detected in strain EC958 are components of defined mobile genetic elements (MGE) that we showed to be absent from the genome of $\mathrm{H} 22$ strain S250. Indeed, we showed that this strain belongs to subclade B1, meaning tothe ancestor of subclone $H 22$ from which evolved the four other $\mathrm{B}$ subclades and subclone $H 30$ with its subclades $\mathrm{C} 1$ and C2. Subclone $H 30$ arose by acquiring, among others, MGEs near which the methyltransferase-encoding genes are located in H30-Rx strain EC958. The three genes identified in strain S250 encode orphan methyltransferases known to be involved in regulation and not methyltransferases involved in restriction modification systems known to inhibit the uptake of non-self DNA and restrict horizontal gene transfer.

We also showed that strain S250 had a gyrA1 allele. This allele was not found in the subclade B1 strains studied by Ben Zakour et al., whereas it was found in some of their strains belonging to subclades B4 and B5 [7]. Concerning our two other fecal $H 22$ strains that harbored the gyrA1a allele, we showed that one belonged to subclade B2 and the other to subclade B4. Plasmid content also varied within our $H 22$ strains: no IncF plasmid in one strain and IncF plasmids with different replicons, F24:B6 and F89:B62, in the two remaining $H 22$ strains, with allele B62 identified here (strain S250) for the first time. Johnson et al. also found plasmids with FII and FIB replicons in most of their $H 22$ strains. However, the two FII and FIB allelic patterns identified by Johnson et al. were different from ours [6]. In contrast, our H30-R strains were all of the same subclade with the same plasmid content: subclade $\mathrm{C} 1$ and the IncF plasmid with the replicon F1:A2:B20This replicon was previously identified in France in CTX-
M-27-producing, fluoroquinolone-resistant $H 30$ strains isolated from stool of children attending different day-care centers in 2012 [14]. It was also very recently shown by Johnson et al. to be the dominant IncF plasmid replicon type among the fluoroquinolone-resistant $H 30$ strains (called H30R1) from different sources whereas replicon F2:A1 was shown to be dominant in those $H 30$ strains producing CTX-M-15 (H30-Rx) [6].

\section{Conclusions}

This study shows, for the first time, that the O25b:H4 ST131 E. coli isolates that were carried in the intestinal microbiota of healthy subjects living in the Paris area in 2006 consisted of strains belonging to both the ancient dominant subclone $H 22$ and the current dominant subclone $H 30$. The genotypic and phenotypic differences identified in this study between these two subclones affect their ability to adapt to the environment. The fecal $H 22$ strains displayed traits increasing their ability to colonize human gut, whereas the fecal H30-R strains displayed traits allowing extra-intestinal adaptation. Thus, resistance to antibiotics may not be the sole advantage of subclone $H 30-\mathrm{R}$ over its precursor subclone $H 22$ in causing worldwide infections.

\section{Additional files}

Additional file 1: Table S1. List of primers designed and used in this study (DOCX $24 \mathrm{~kb}$ )

Additional file 2: Table S2. Escherichia coli virulence factor encoding genes tested by the PCR method. (DOCX $25 \mathrm{~kb}$ )

Additional file 3: Table S3. Distribution and identity percentage of the genes previously shown to be involved in biofilm formation [28] in representatives of different ST131 H subclones. (DOCX $41 \mathrm{~kb}$ )

Additional file 4: Table S4. $\mathrm{H} 22$ and $\mathrm{H} 30$ strains with a whole genome sequence available in NCBI databases either under a circular DNA form or a fragmented DNA form. (DOCX $16 \mathrm{~kb}$ )

\section{Abbreviations}

BFI: Biofilm index; BLAST: Basic Local Alignment Search Tool; bp: Base pair; ESBL: Extended-spectrum $\beta$-lactamase; ExPEC: Extra-intestinal pathogenic $E$. coli; $M_{1}$ : Mono-mannose; $M_{3}$ : Tri-mannose; MGE: Mobile genetic elements; NCBI: National Center for Biotechnology Information; nt: nucleotide; QRDR: Quinolone-resistance determining region; SNP: Single-nucleotide polymorphism; ST: Sequence type; VF: Virulence factor; VFDB: Virulence factor database; WGS: Whole genome shotgun

\section{Acknowledgments}

We thank Bill Hempel for his contribution in the writing of the MS in English.

\section{Funding}

This work was partially supported by grants AGL2013-47852-R (Ministerio de Economía y Competitividad, Gobierno de España), CN2012/303 and EM2014/ 001 (Consellería de Cultura, Educación e Ordenación Universitaria, Xunta de Galicia and The European Regional Development Fund, ERDF), and RCT-2012-02 (Direction Générale de l'Offre de Soins et Institut National de la Santé et de la Recherche Médicale, Paris, France).

Availability of data and materials

The whole genome shotgun (WGS) project of $\mathrm{H} 22$ strain $\mathrm{S} 250$ is available in the [DDBJ/EMBL/GenBank] repositories under the accession LXQL00000000 
[https://www.ncbi.nlm.nih.gov/assembly/GCF_001648715.1/]. Genome annotation was performed using the NCBI Prokaryotic Genome Annotation Pipeline. The version described in this paper is version LXQL01000000.

\section{Authors' contributions}

MHNC conceived the study, supervised the research, and drafted the manuscript. MP performed the genomic analyses. AM, NM, JPL, OB, and VLB performed the molecular and bacterial experiments. JB participated in the design of the molecular experiments and analyzed the data. DH supervised and participated in the genomic analyses and revised the manuscript. All authors read and approved the final manuscript.

\section{Competing interests}

The authors declare that they have no competing interests.

\section{Consent for publication}

Not applicable.

\section{Ethics approval and consent to participle}

Not applicable.

\section{Publisher's Note}

Springer Nature remains neutral with regard to jurisdictional claims in published maps and institutional affiliations.

\section{Author details \\ ${ }^{1}$ Service de Microbiologie, Hôpital Beaujon, AP-HP, Clichy, France. ${ }^{2}$ Faculté de Médecine Paris Diderot, Paris, France. ${ }^{3}$ INSERM UMR 1137, Université Paris 7 , Paris, France. ${ }^{4}$ Laboratoire d'Hygiène Hospitalière, CHRU Besançon, Besançon, France. ${ }^{5}$ UMR 6249 Chrono-Environnement, Besançon, France. ${ }^{6}$ Laboratorio de Referencia de E. coli (LREC), Facultad de Veterinaria, Universidad de Santiago de Compostela, Lugo, Spain. ${ }^{7}$ Institut National de la Santé et de la Recherche Médicale, U1047, Université de Montpellier, UFR de Médecine, Nîmes, France. ${ }^{8}$ Service de Microbiologie, CHU Carémeau, Nîmes, France. ${ }^{9}$ Laboratoire d'Analyse Médicale du Centre, Coulommiers, France.}

\section{Received: 19 November 2016 Accepted: 14 March 2017} Published online: 27 March 2017

\section{References}

1. Nicolas-Chanoine MH, Bertrand X, Madec JY. Escherichia coli ST131, an intriguing clonal group. Clin Microbiol Rev. 2014;27:543-74.

2. Price LB, Johnson JR, Aziz M, Clabots C, Johnston B, Tchesnokova V, Nordstrom L, Billig M, Chattopadhyay S, et al. The epidemic of extendedspectrum- $\beta$-lactamase-producing Escherichia coli ST131 is driven by a single highly pathogenic subclone, H30-Rx. MBio. 2013;4:1-11.

3. Johnson JR, Tchesnokova V, Johnston B, Clabots C, Roberts PL, Billig M, Riddell K, Rogers P, Qin X, et al. Abrupt emergence of a single dominant multidrug-resistant strain of Escherichia coli. J Infect Dis. 2013;207:919-28.

4. Olesen B, Frimodt-Moller J, Leihof RF, Struve C, Johnston B, Hansen DS, Scheutz F, Krogfelt KA, Kuskowski MA, et al. Temporal trends in antimicrobial resistance and virulence-associated traits within the Escherichia coli sequence type 131 clonal group and its H30 and H30-Rx subclones, 1968 to 2012. Antimicrob Agents Chemother. 2014;58:6886-95.

5. Stoesser N, Sheppard AE, Pankhurst L, De Maio N, Moore CE, Sebra R, Turner $P$, Anson LW, Kasarskis A, et al. Evolutionary history of the gobal emergence of the Escherichia coli epidemic clone ST131. MBio. 2016;7:e02162.

6. Johnson TJ, Danzeisen JL, Youmans B, Case K, Llop K, Munoz-Aguayo J, Flores-Figueroa C, Aziz M, Stoesser N, et al. Separate F-type plasmids have shaped the evolution of the H30 suclone of Escherichia coli sequence type 131. mSphere. 2016;1:1-15.

7. Ben Zakour NL, Alsheikh-Hussain AS, Ashcroft MM, Khanh Nhu NT, Roberts LW, Stanton-Cook M, Schembri MA, Beatson SA. Sequential acquisition of virulence and fluoroquinolone resistance has shaped the evolution of Escherichia coli ST131. MBio. 2016;7:e00347-16.

8. Can F, Kurt-Azap O, Ispir P, Nurtop E, Seref C, Loclar I, Aktas ON, Orhan YC, Ergonul O. The clinical impact of ST131 H30-Rx subclone in urinary tract infections due to multidrug-resistant Escherichia coli. J Glob Antimicrob Resist. 2016;4:49-52.

9. Rodrigues C, Machado E, Fernandes S, Peixe L, Novais A. An update on faecal carriage of ESBL-producing Enterobacteriaceae by Portuguese healthy humans: detection of the H30 subclone of B2-ST131 Escherichia coli producing CTX-M-27. J Antimicrob Chemother. 2016;71:1120-2.

10. Ranjan A, Shaik S, Hussain A, Nandanwar N, Semmler T, Jadhav S, Wieler LH, Ahmed N. Genomic and functional portrait of a highly virulent, CTX-M-15producing H30-Rx subclone of Escherichia coli sequence type 131. Antimicrob Agents Chemother. 2015;59:6087-95.

11. Johnson JR, Johnston B, Kuskowski MA, Sokurenko EV, Tchesnokova V. Intensity and mechanisms of fluoroquinolone resistance within the $\mathrm{H} 30$ and H30Rx subclones of Escherichia coli sequence type 131 compared with other fluoroquinolone-resistant E. coli. Antimicrob Agents Chemother. 2015:59:4471-80.

12. Dahbi G, Mora A, Mamani R, Lopez C, Alonso MP, Marzoa J, Blanco M, Herrera A, Viso S, et al. Molecular epidemiology and virulence of Escherichia coli O16:H5-ST131: comparison with H30 and H30-Rx subclones of O25b:H4ST131. Int J Med Microbiol. 2014;304:1247-57.

13. Rogers BA, Ingram PR, Runnegar N, Pitman MC, Freeman JT, Athan E, Havers S, Sidjabat HE, Gunning E, et al. Sequence type 131 fimH30 and fimH41 subclones amongst Escherichia coli isolates in Australia and New Zealand. Int J Antimicrob Agents. 2015;45:351-8.

14. Blanc V, Leflon-Guibout V, Blanco J, Haenni M, Madec JY, Rafignon G, Bruno P, Mora A, Lopez C, et al. Prevalence of day-care centre children (France) with faecal CTX-M-producing Escherichia coli comprising O25b:H4 and 016: H5 ST131 strains. J Antimicrob Chemother. 2014;69:1231-7.

15. Zhong YM, Liu WE, Liang XH, Li YM, Jian ZJ, Hawkey PM. Emergence and spread of O16-ST131 and O25b-ST131 clones among faecal CTX-Mproducing Escherichia coli in healthy individuals in Hunan Province. China J Antimicrob Chemother. 2015;70:2223-7.

16. Gurnee EA, Ndao IM, Johnson JR, Johnston BD, Gonzalez MD, Burnham CA, Hall-Moore CM, McGhee JE, Mellmann A, et al. Gut colonization of healthy children and their mothers with pathogenic ciprofloxacin-resistant Escherichia coli. J Infect Dis. 2015;212:1862-8.

17. Mohamed M, Clabots C, Porter SB, Thuras P, Johnson JR. Isolation and characterization of Escherichia coli sequence type 131 (ST131) and other antimicrobial-resistant gram-negative bacilli from clinical stool samples from veterans. Antimicrob Agents Chemother. 2016;60:4638-45.

18. Leflon-Guibout V, Blanco J, Amaqdouf K, Mora A, Guize L, Nicolas-Chanoine $\mathrm{MH}$. Absence of CTX-M enzymes but high prevalence of clones, including clone ST131, among fecal Escherichia coli isolates from healthy subjects living in the area of Paris. France J Clin Microbiol. 2008;46:3900-5.

19. Lavigne JP, Vergunst AC, Goret L, Sotto A, Combescure C, Blanco J, O'Callaghan D, Nicolas-Chanoine MH. Virulence potential and genomic mapping of the worldwide clone Escherichia coli ST131. PLoS One. 2012;7:e34294.

20. Weissman SJ, Johnson JR, Tchesnokova V, Billig M, Dykhuizen D, Riddell K, Rogers P, Qin X, Butler-Wu S, et al. High-resolution two-locus clonal typing of extraintestinal pathogenic Escherichia coli. Appl Environ Microbiol. 2012;78:1353-60.

21. Leflon-Guibout V, Jurand C, Bonacorsi S, Espinasse F, Guelfi MC, Duportail F, Heym B, Bingen E, Nicolas-Chanoine MH. Emergence and spread of three clonally related virulent isolates of CTX-M-15-producing Escherichia coli with variable resistance to aminoglycosides and tetracycline in a French geriatric hospital. Antimicrob Agents Chemother. 2004;48:3736-42.

22. Guillard T, de Champs C, Moret H, Bertrand X, Scheftel JM, Cambau E. Highresolution melting analysis for rapid characterization of qnr alleles in clinical isolates and detection of two novel alleles, qnrB25 and qnrB42. J Antimicrob Chemother. 2012;67:2635-9.

23. Paltansing S, Kraakman ME, Ras JM, Wessels E, Bernards AT. Characterization of fluoroquinolone and cephalosporin resistance mechanisms in Enterobacteriaceae isolated in a Dutch teaching hospital reveals the presence of an Escherichia coli ST131 clone with a specific mutation in parE. J Antimicrob Chemother. 2013;68:40-5.

24. Criscuolo A, Brisse S. AlienTrimmer: a tool to quickly and accurately trim off multiple short contaminant sequences from high-throughput sequencing reads. Genomics. 2013;102:500-6.

25. Rissman Al, Mau B, Biehl BS, Darling AE, Glasner JD, Perna NT. Reordering contigs of draft genomes using the mauve aligner. Bioinformatics. 2009;25:2071-3.

26. Hyatt D, Chen GL, Locascio PF, Land ML, Larimer FW, Hauser LJ. Prodigal: prokaryotic gene recognition and translation initiation site identification. BMC Bioinf. 2010;11:119.

27. Li W, Jaroszewski L, Godzik A. Clustering of highly homologous sequences to reduce the size of large protein databases. Bioinformatics. 2001;17:282-3. 
28. Niba ET, Naka Y, Nagase M, Mori H, Kitakawa M. A genome-wide approach to identify the genes involved in biofilm formation in E. coli. DNA Res. 2007;14:237-46.

29. Maltby R, Leatham-Jensen MP, Gibson T, Cohen PS, Conway T. Nutritional basis for colonization resistance by human commensal Escherichia coli strains HS and Nissle 1917 against E. coli O157:H7 in the mouse intestine. PLoS One. 2013:8:e53957.

30. Gomez KM, Rodriguez A, Rodriguez Y, Ramirez AH, Isturiz T. The subsidiary Gntll system for gluconate metabolism in Escherichia coli: alternative induction of the gntV gene. Biol Res. 2011;44:269-75.

31. Forde BM, Phan MD, Gawthorne JA, Ashcroft MM, Stanton-Cook M, Sarkar S, Peters KM, Chan KG, Chong TM, et al. Lineage-specific methyltransferases define the methylome of the globally disseminated Escherichia coli ST131 clone. MBio. 2015;6:e01602-15.

32. Carattoli A, Zankari E, Garcia-Fernandez A, Voldby Larsen M, Lund O, Villa L, Moller Aarestrup F, Hasman H. In silico detection and typing of plasmids using PlasmidFinder and plasmid multilocus sequence typing. Antimicrob Agents Chemother. 2014;58:3895-903.

33. Johnson JR, Stell AL. Extended virulence genotypes of Escherichia coli strains from patients with urosepsis in relation to phylogeny and host compromise. J Infect Dis. 2000;181:261-72.

34. Blanco J, Mora A, Mamani R, Lopez C, Blanco M, Dahbi G, Herrera A, Marzoa $J$, Fernandez $V$, et al. Four main virotypes among extended-spectrum- $\beta$ lactamase-producing isolates of Escherichia coli O25b:H4-B2-ST131: bacterial, epidemiological, and clinical characteristics. J Clin Microbiol. 2013;51:3358-67.

35. Blanco M, Alonso MP, Nicolas-Chanoine MH, Dahbi G, Mora A, Blanco JE, Lopez C, Cortes P, Llagostera M, et al. Molecular epidemiology of Escherichia coli producing extended-spectrum $\beta$-lactamases in Lugo (Spain): dissemination of clone O25b:H4-ST131 producing CTX-M-15. J Antimicrob Chemother. 2009;63:1135-41.

36. Sokurenko EV, Courtney HS, Maslow J, Siitonen A, Hasty DL. Quantitative differences in adhesiveness of type 1 fimbriated Escherichia coli due to structural differences in fimH genes. J Bacteriol. 1995;177:3680-6.

37. Sokurenko EV, Chesnokova V, Dykhuizen DE, Ofek I, Wu XR, Krogfelt KA, Struve C, Schembri MA, Hasty DL. Pathogenic adaptation of Escherichia coli by natural variation of the FimH adhesin. Proc Natl Acad Sci U S A. 1998:95:8922-6.

38. Leatham MP, Stevenson SJ, Gauger EJ, Krogfelt KA, Lins JJ, Haddock TL, Autieri SM, Conway T, Cohen PS. Mouse intestine selects nonmotile flhDC mutants of Escherichia coli MG1655 with increased colonizing ability and better utilization of carbon sources. Infect Immun. 2005;73:8039-49.

39. D'Argenio DA, Wu M, Hoffman LR, Kulasekara HD, Deziel E, Smith EE, Nguyen H, Ernst RK, Larson Freeman TJ, et al. Growth phenotypes of Pseudomonas aeruginosa lasR mutants adapted to the airways of cystic fibrosis patients. Mol Microbiol. 2007;64:512-33.

40. Chavant P, Gaillard-Martinie B, Talon R, Hebraud M, Bernardi T. A new device for rapid evaluation of biofilm formation potential by bacteria. J Microbiol Methods. 2007:68:605-12.

41. Totsika M, Beatson SA, Sarkar S, Phan MD, Petty NK, Bachmann N, Szubert $\mathrm{M}$, Sidjabat HE, Paterson DL, et al. Insights into a multidrug resistant Escherichia coli pathogen of the globally disseminated ST131 lineage: genome analysis and virulence mechanisms. PLoS One. 2011;6:e26578.

42. Pantel A, Dunyach-Remy C, Ngba Essebe C, Mesureur J, Sotto A, Pages JM, Nicolas-Chanoine $\mathrm{MH}$, Lavigne JP. Modulation of membrane influx and efflux in Escherichia coli sequence type 131 has an impact on bacterial motility, biofilm formation, and virulence in a Caenorhabditis elegans model. Antimicrob Agents Chemother. 2016;60:2901-11.

43. Owens RCJ, Johnson JR, Stogsdill P, Yarmus L, Lolans K, Quinn J. Community transmission in the United States of a CTX-M-15-producing sequence type ST131 Escherichia coli strain resulting in death. J Clin Microbiol. 2011:49:3406-8.

44. Hussain A, Ranjan A, Nandanwar N, Babbar A, Jadhav S, Ahmed N. Genotypic and phenotypic profiles of Escherichia coli isolates belonging to clinical sequence type 131 (ST131), clinical non-ST131, and fecal non-ST131 lineages from India. Antimicrob Agents Chemother. 2014:58:7240-9.

45. Morgand M, Vimont S, Bleibtreu A, Boyd A, Thien HV, Zahar JR, Denamur E, Arlet G. Extended-spectrum $\beta$-lactamase-producing Escherichia coli infections in children: are community-acquired strains different from nosocomial strains? Int J Med Microbiol. 2014;304:970-6.

46. Sarkar S, Vagenas D, Schembri MA, Totsika M. Biofilm formation by multidrug resistant Escherichia coli ST131 is dependent on type 1 fimbriae and assay conditions. Pathog Dis. 2016;74(3):ftw013
47. Peekhaus N, Conway T. What's for dinner?: Entner-Doudoroff metabolism in Escherichia coli. J Bacteriol. 1998:180:3495-502.

48. Chen SL, Hung CS, Pinkner JS, Walker JN, Cusumano CK, Li Z, Bouckaert J, Gordon Jl, Hultgren SJ. Positive selection identifies an in vivo role for FimH during urinary tract infection in addition to mannose binding. Proc Natl Acad Sci U S A. 2009;106:22439-44.

49. Conway T, Cohen PS. Commensal and pathogenic Escherichia coli metabolism in the gut. Microbiol Spectr. 2015:3:1-15.

\section{Submit your next manuscript to BioMed Central and we will help you at every step:}

- We accept pre-submission inquiries

- Our selector tool helps you to find the most relevant journal

- We provide round the clock customer support

- Convenient online submission

- Thorough peer review

- Inclusion in PubMed and all major indexing services

- Maximum visibility for your research

Submit your manuscript at www.biomedcentral.com/submit
) Biomed Central 